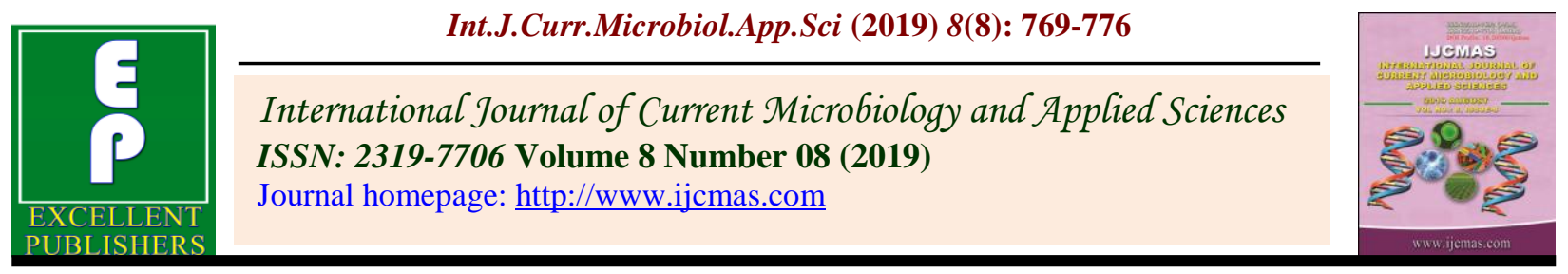

Original Research Article https://doi.org/10.20546/ijcmas.2019.808.087

\title{
Identification of Promising Clones of Potato for Resistance against Leaf Curl Virus
}

\author{
Nirmodh Prabha*, H.C. Nanda and Suresh Kumar Sharma
}

Department of Genetics and Plant Breeding, SantKabir College of Agriculture and Research Station, IGKV, Kawardha, Kabirdham, 491995. (C.G.) India

*Corresponding author

\section{A B S T R A C T}

Studies on screening and evaluation of clonal progenies of potato (Solanum

Keywords

Potato (Solanum tuberosum L.), Genetics and plant breeding

Article Info

Accepted: 07 July 2019 Available Online:

10 August 2019 tuberosum L.) for resistance against leaf curl virus (LCV) diseases were conducted under AICRP on Potato, Department of Genetics and Plant Breeding, College of Agriculture, Indira Gandhi Krishi Vishwavidyalaya, Raipur (C.G.). In present investigation, Screening against leaf curl virus revealed that most of the genotypes showed moderate resistance against leaf curl virus, except 14 genotypes (CIP 398203-2-1, CIP 304014-2-1 and KufriPukhraj of $\mathrm{F}_{1} \mathrm{C}_{2}$ progenies and CIP 398203-2-2-1, CIP 302431-4-1-1, CIP 304012-1-4-1, CIP 304012-6-2-3, CIP 303405-7-4-1, CIP 303405-9-11, CIP 304146-11-2-2, CIP 304014-1-3-1, CIP 304014-12-2-1, CIP 398181-10-1-1, CIP 304124-7-2-1 of $\mathrm{F}_{1} \mathrm{C}_{3}$ ) which showed highly resistance reaction against leaf curl virus. These resistant genotypes can be exploited for developing high yielding and disease resistant varieties in potato.

\section{Introduction}

Potato is an important horticultural product used by both rich and poor. Since potato gives an exceptionally high yield, produces more edible energy and protein per unit area and time than many other food crops, it is ideal which can grow well in multiple cropping systems prevalent in both types of countries having tropical and subtropical agro-climatic conditions. Potato is a valuable food for those who seek to lower their blood pressure. Further, there is no truth in the general belief that potato promotes fat accumulation (PushkarNath, 1976). The potato crop is afflicted by a number of fungal, bacterial and viral diseases as well as insect-pests. However, leaf curl virus diseases have become serious problem in potato, which affect the crop growth and production. Garg et al., (2001) reported a new disease on potato, which produced para crinkle symptoms and the potato plants infected with PALCV showed chlorotic blotching, crinkling, mosaic, apical leaf curling and stunting. Severe yield losses due to this disease have been reported 
in potato. Annual loss due to potato viruses with an average of $30-40 \%$ incidence cause about 25- $30 \%$ yield reduction Khurana (1999). Lakra (2002) reported that with 100 per cent disease incidence of PALCV, more than 50 per cent losses in yield has been reported in early sown crop of potato cultivar KufriAshoka. The most deleterious effect was observed on reduction in leaf area, chlorophyll content, plant height, number of stems per plant, number of tubers per plant and weight of tubers per plant Lakra (2003a). The indiscriminate use of insecticide results in not only environmental pollutions but is also responsible for so many health hazards. Host resistance plays a vital role in its management. A large number of varieties having medium to high level of early resistance have been developed, however, development of matching virulence in pathogens had eroded many varieties from the cultivation. Therefore, breeding for resistance to Leaf Curl Virus continual activity of crop improvement. Therefore, in view of the importance of crop and disease, the present investigation was planned.

\section{Materials and Methods}

The experiment were consisted of 18 clonal bulks, $43 \mathrm{~F}_{1} \mathrm{C}_{2}$ and $90 \mathrm{~F}_{1} \mathrm{C}_{3}$ clonal progenies of potato along with four check varieties (Kufri Jawahar, Kufri Pukhraj, Kufri Ashoka and Kufri Surya) obtained from genetic stock of AICRP on potato, Department of Genetics and Plant Breeding, COA, IGKV, Raipur (C.G.) grown in randomized complete block design with three replications. All potato entries were screened against leaf curl virus under natural field conditions (Table 1). Observations on disease incidence were recorded and calculated by following formula:

Total number of infected plants Disease incidence $(\%)=$--------------- x 100

Total number of plants

\section{Results and Discussion}

Screening of bulks, $F_{1} C_{2}$ and $F_{1} C_{3}$ clonal progenies of potato against leaf curl virus

A set of 18 selected clones bulk population was screened against leaf curl virus disease. The per cent disease incidence transformed into square root and presented in (Table 2). The minimum virus incidence was observed in KufriAshoka (1.10\%) and Kufri Surya (1.10\%) followed by CIP 398203 (2.20\%) and KufriPukhraj (3.30\%). The maximum leaf curl virus was observed in CIP 304014 (10.0\%) followed by CIP $304102 \quad(8.90 \%)$, KufriJawahar (8.90\%), CIP 302024 (7.80\%), CIP 302431 (6.70\%), CIP 304146 (6.70\%) and CIP $398181(6.70 \%)$.

In $\mathrm{F}_{1} \mathrm{C}_{2}$ clonal progenies data (Table 3) revealed that, the entries CIP 398203-2-1, CIP 304014-2-1 and KufriPukhraj (check) had nil (0\%) incidence of leaf curl virus. This was followed by CIP 398203-2-2, CIP 398203- 31, CIP 302431-8-1, CIP 303405-11-1, CIP 303405-15-1, CIP 303408-9-2, CIP 30410217-1, CIP 398201-19-1, CIP 304014-1-1, CIP 304124-14-1, CIP 304124-15-1, KufriAshoka and Kufri Surya all showed 3.30 per cent incidence. Whereas, the maximum disease incidence was noticed to be 26.70 per cent in entries CIP 304012-1-2 and CIP 304146-1-1 followed by 23.30 per cent incidence observed for CIP 304146-2-1, CIP 304146-3-1 and CIP 398068-22-1

Similarly, in $\mathrm{F}_{1} \mathrm{C}_{3}$ clonal progenies the data (Table 4) showed CIP 398203-2-2-1, CIP 302431-4-1-1, CIP 304012-1-4-1, CIP 304012-6-2-3, CIP 303405-7-4-1, CIP 303405-9-1-1, CIP 304146-11-2-2, CIP 304014-1-3-1, CIP 304014-12-2-1, CIP 398181-10-1-1 and CIP 304124-7-2-1 all had zero $(0 \%)$ incidence of leaf curl virus. Similarly, 3.30 per cent incidence was recorded in CIP 398203-4-1-1, CIP 302431-2- 
1-1, CIP 304012-6-1-1, CIP 303405-5-3-1, CIP 303408-7-3-1, CIP 304146-11-3-1, CIP 304146-2-2-1, CIP 398201-11-6-1, CIP 304014-2-6-1, CIP 398181-17-2-1, CIP 304124-2-5-1, CIP 303139-4-4-1, CI 3031392-2-1, CIP 303139-10-3-1, CIP 398068-3-2-1,
CIP 398068-9-4-1, Kufri Pukhraj and Kufri Surya. While, the maximum incidence was recorded in entries viz., CIP 398201-15-4-1 (30.0\%), CIP 398181-9-3-1 (26.70\%), CIP 302024-3-4-2 (23.30\%) and CIP 304102-4-2-1 (23.30\%).

Table.1 Categorization of varieties on the basis of per cent disease incidence in leaf curl virus grouped in 1-9 scale (AICRP, Potato)

\begin{tabular}{|c|l|l|}
\hline S. No. & \multicolumn{1}{|c|}{ Per cent infection } & \multicolumn{1}{|c|}{ Category } \\
\hline 1 & No infection & Highly resistant \\
\hline 2 & Less infection & Very resistant \\
\hline 3 & $0.1-1.0$ & Resistant \\
\hline 4 & $1.1-10.0$ & Moderately resistant \\
\hline 5 & $10.1-20.0$ & Slightly resistant \\
\hline 6 & $20.1-30.0$ & Scarcely resistant \\
\hline 7 & $30.1-40.0$ & Susceptible \\
\hline 8 & $40.1-50.0$ & Very susceptible \\
\hline 9 & above 50.0 & Extremely susceptible \\
\hline
\end{tabular}

Table.2 Reaction and summary of potato bulks against leaf curl virus disease

\begin{tabular}{|c|c|c|c|c|c|c|c|}
\hline $\begin{array}{l}\text { S. } \\
\text { No. }\end{array}$ & $\begin{array}{c}\text { Entries } \\
\text { (Potato bulks) }\end{array}$ & $\begin{array}{l}\text { Leaf curl virus } \\
\text { incidence }(\%)\end{array}$ & Summary & $\begin{array}{l}\text { S. } \\
\text { No. }\end{array}$ & $\begin{array}{c}\text { Entries } \\
\text { (Potato bulks) }\end{array}$ & $\begin{array}{l}\text { Leaf curl virus } \\
\text { incidence }(\%)\end{array}$ & Summary \\
\hline \multirow[t]{2}{*}{1} & CIP 398203 & 2.20 & MR & 10 & CIP 304014 & 10.00 & MR \\
\hline & & $(1.53)$ & & & & $(3.21)$ & \\
\hline \multirow[t]{2}{*}{2} & CIP 302024 & 7.80 & MR & 11 & CIP 398181 & 6.70 & MR \\
\hline & & $(2.80)$ & & & & $(2.68)$ & \\
\hline \multirow[t]{2}{*}{3} & CIP 302431 & 6.70 & MR & 12 & CIP 304124 & 4.40 & MR \\
\hline & & $(2.37)$ & & & & $(2.19)$ & \\
\hline \multirow[t]{2}{*}{4} & CIP 304012 & 4.50 & MR & 13 & CIP 303139 & 5.60 & MR \\
\hline & & $(2.02)$ & & & & $(2.44)$ & \\
\hline \multirow[t]{2}{*}{5} & CIP 303405 & 5.60 & MR & 14 & CIP 398068 & 4.40 & MR \\
\hline & & $(2.21)$ & & & & $(2.19)$ & \\
\hline \multirow[t]{2}{*}{6} & CIP 303408 & 5.50 & MR & 15 & KufriJawahar & 8.90 & MR \\
\hline & & $(2.38)$ & & & & $(2.97)$ & \\
\hline \multirow[t]{2}{*}{7} & CIP 304102 & 8.90 & MR & 16 & KufriAshoka & 1.10 & MR \\
\hline & & (2.97) & & & & $(1.12)$ & \\
\hline \multirow[t]{2}{*}{8} & CIP 304146 & 6.70 & MR & 17 & KufriPukhraj & 3.30 & MR \\
\hline & & $(2.62)$ & & & & $(1.95)$ & \\
\hline \multirow[t]{2}{*}{9} & CIP 398201 & 4.50 & MR & 18 & Kufri Surya & 1.10 & MR \\
\hline & & $(3.87)$ & & & & $(1.12)$ & \\
\hline \multicolumn{8}{|c|}{$\begin{array}{c}\text { SEm+ } 0.714 \\
\text { C.D. (at 5\%) NS }\end{array}$} \\
\hline \multicolumn{8}{|c|}{ Average of three replications } \\
\hline \multirow{2}{*}{\multicolumn{2}{|c|}{$\begin{array}{l}\mathrm{MR}=\text { Moderately resistant } \\
\mathrm{SR}=\text { Slightly resistant }\end{array}$}} & $\mathrm{MS}=\mathrm{M}$ & rately susce & ible & $\mathrm{HR}=$ Highly $\mathrm{r}$ & $\operatorname{tant}$ & \\
\hline & & $\mathrm{S}=\mathrm{Susc}$ & tible & & $\mathrm{ScR}=$ Scarcely & sistant & \\
\hline
\end{tabular}


Table.3 Reaction and summary of $\mathrm{F}_{1} \mathrm{C}_{2}$ clonal progenies against leaf curl virus

\begin{tabular}{|c|c|c|c|c|c|c|c|}
\hline $\begin{array}{l}\text { S. } \\
\text { No. }\end{array}$ & $\begin{array}{c}\text { Entries } \\
\left(\mathrm{F}_{1} \mathrm{C}_{2} \text { progenies }\right)\end{array}$ & $\begin{array}{l}\text { Leaf curl virus } \\
\text { incidence }(\%)\end{array}$ & Summary & S. No. & $\begin{array}{c}\text { Entries } \\
\left(\mathrm{F}_{1} \mathrm{C}_{2} \text { progenies }\right)\end{array}$ & $\begin{array}{l}\text { Leaf curl virus } \\
\text { incidence }(\%)\end{array}$ & Summary \\
\hline \multirow[t]{2}{*}{1} & CIP 398203-2-1 & 0.00 & MR & 23 & CIP 398201-19-1 & 3.30 & MR \\
\hline & & $(0.57)$ & & & & $(6.52)$ & \\
\hline \multirow[t]{2}{*}{2} & CIP 398203-2-2 & 3.30 & MR & 24 & CIP 398201-19-2 & 10.00 & MR \\
\hline & & (6.52) & & & & (15.18) & \\
\hline \multirow[t]{2}{*}{3} & CIP 398203-3-1 & 3.30 & MR & 25 & CIP 398201-20-1 & 10.00 & MR \\
\hline & & $(6.52)$ & & & & (15.18) & \\
\hline \multirow[t]{2}{*}{4} & CIP 302024-10-1 & 13.30 & SR & 26 & CIP 398201-22-1 & 10.00 & MR \\
\hline & & (21.14) & & & & (18.43) & \\
\hline \multirow[t]{2}{*}{5} & CIP 302024-11-1 & 6.70 & MR & 27 & CIP 304014-1-1 & 3.30 & MR \\
\hline & & (12.48) & & & & $(6.52)$ & \\
\hline \multirow[t]{2}{*}{6} & CIP 302024-11-2 & 10.00 & MR & 28 & CIP 304014-2-1 & 0.00 & HR \\
\hline & & $(15.10)$ & & & & $(0.57)$ & \\
\hline \multirow[t]{2}{*}{7} & CIP 302431-8-1 & 3.30 & MR & 29 & CIP 398181-1-1 & 13.30 & SR \\
\hline & & $(6.52)$ & & & & (21.14) & \\
\hline \multirow[t]{2}{*}{8} & CIP 302431-9-1 & 10.00 & MR & 30 & CIP 398181-2-1 & 6.70 & MR \\
\hline & & (15.18) & & & & $(9.23)$ & \\
\hline \multirow[t]{2}{*}{9} & CIP 304012-1-1 & 13.30 & SR & 31 & CIP 304124-14-1 & 3.30 & MR \\
\hline & & (21.14) & & & & $(6.52)$ & \\
\hline \multirow[t]{2}{*}{10} & CIP 304012-1-2 & 26.70 & $\mathrm{ScR}$ & 32 & CIP 304124-15-1 & 3.30 & MR \\
\hline & & $(30.98)$ & & & & $(6.52)$ & \\
\hline \multirow[t]{2}{*}{11} & CIP 303405-11-1 & 3.30 & MR & 33 & CIP 303139-11-1 & 10.00 & MR \\
\hline & & $(6.52)$ & & & & (15.18) & \\
\hline \multirow[t]{2}{*}{12} & CIP 303405-13-1 & 6.70 & MR & 34 & CIP 303139-12-1 & 20.00 & SR \\
\hline & & (12.48) & & & & (26.06) & \\
\hline \multirow[t]{2}{*}{13} & CIP 303405-15-1 & 3.30 & MR & 35 & CIP 303139-13-1 & 10.00 & MR \\
\hline & & $(6.52)$ & & & & (15.18) & \\
\hline \multirow[t]{2}{*}{14} & CIP 303408-9-1 & 6.70 & MR & 36 & CIP 398068-19-1 & 20.00 & SR \\
\hline & & (12.48) & & & & (26.06) & \\
\hline \multirow[t]{2}{*}{15} & CIP 303408-9-2 & 3.30 & MR & 37 & CIP 398068-21-1 & 13.30 & SR \\
\hline & & (6.52) & & & & (21.14) & \\
\hline \multirow[t]{2}{*}{16} & CIP 303408-10-1 & 10.00 & MR & 38 & CIP 398068-21-2 & 10.00 & MR \\
\hline & & (15.18) & & & & (15.18) & \\
\hline \multirow[t]{2}{*}{17} & CIP 304102-15-1 & 6.70 & MR & 39 & CIP 398068-22-1 & 23.30 & ScR \\
\hline & & (12.48) & & & & (28.06) & \\
\hline \multirow[t]{2}{*}{18} & CIP 304102-16-1 & 10.00 & MR & 40 & KufriJawahar & 16.70 & SR \\
\hline & & $(18.43)$ & & & & $(23.84)$ & \\
\hline \multirow[t]{2}{*}{19} & CIP 304102-17-1 & 3.30 & MR & 41 & KufriAshoka & 3.30 & MR \\
\hline & & $(6.52)$ & & & & $(6.52)$ & \\
\hline \multirow[t]{2}{*}{20} & CIP 304146-1-1 & 26.70 & ScR & 42 & KufriPukhraj & 0.00 & HR \\
\hline & & (30.98) & & & & $(0.57)$ & \\
\hline \multirow[t]{2}{*}{21} & CIP 304146-2-1 & 23.30 & $\mathrm{ScR}$ & 43 & Kufri Surya & 3.30 & MR \\
\hline & & (28.77) & & & & $(6.52)$ & \\
\hline \multirow[t]{4}{*}{22} & CIP 304146-3-1 & 23.30 & $\mathrm{ScR}$ & & & & \\
\hline & & $(28.77)$ & & & & & \\
\hline & & & & & $\mathbf{S E m}_{\underline{ \pm}}$ & 5.520 & \\
\hline & & & & & C.D. (at 5\%) & 15.51 & \\
\hline
\end{tabular}

Average of three replications

$\mathrm{MR}=$ Moderately resistant

$\mathrm{SR}=$ Slightly resistant
Data in parenthesis are transformed value)

MS=Moderately susceptible

$\mathrm{S}=$ Susceptible
$\mathrm{HR}=$ Highly resistant

$\mathrm{ScR}=$ Scarcely resistant 
Table.4 Reaction and summary of $\mathrm{F}_{1} \mathrm{C}_{3}$ clonal progenies against leaf curl virus

\begin{tabular}{|c|c|c|c|c|c|c|c|}
\hline $\begin{array}{l}\text { S. } \\
\text { No. }\end{array}$ & $\begin{array}{c}\text { Entries } \\
\left(\mathrm{F}_{1} \mathrm{C}_{3} \text { progenies }\right)\end{array}$ & $\begin{array}{c}\text { Leaf curl virus } \\
\text { incidence }(\%)\end{array}$ & Summary & $\begin{array}{l}\text { S. } \\
\text { No. }\end{array}$ & $\begin{array}{c}\text { Entries } \\
\left(\mathrm{F}_{1} \mathrm{C}_{3} \text { progenies }\right)\end{array}$ & $\begin{array}{c}\text { Leaf curl virus } \\
\text { incidence }(\%)\end{array}$ & Summary \\
\hline \multirow[t]{2}{*}{1} & CIP 398203-2-2-1 & 0.00 & HR & 46 & CIP 304146-11-2-2 & 0.00 & HR \\
\hline & & $(0.57)$ & & & & $(0.57)$ & \\
\hline \multirow[t]{2}{*}{2} & CIP 398203-4-1-1 & 3.30 & MR & 47 & CIP 304146-11-3-1 & 3.30 & MR \\
\hline & & $(6.52)$ & & & & $(6.52)$ & \\
\hline \multirow[t]{2}{*}{3} & CIP 398203-4-1-2 & 6.70 & MR & 48 & CIP 304146-1-4-1 & 6.70 & MR \\
\hline & & (12.48) & & & & $(12.48)$ & \\
\hline \multirow[t]{2}{*}{4} & CIP 398203-5-3-1 & 10.00 & MR & 49 & CIP 304146-2-2-1 & 3.30 & MR \\
\hline & & $(18.43)$ & & & & $(6.52)$ & \\
\hline \multirow[t]{2}{*}{5} & CIP 398203-6-1-3 & 13.30 & SR & 50 & CIP 398201-3-3-1 & 10.00 & MR \\
\hline & & $(17.89)$ & & & & $(18.43)$ & \\
\hline \multirow[t]{2}{*}{6} & CIP 398203-7-4-1 & 6.70 & MR & 51 & CIP 398201-3-3-2 & 10.00 & MR \\
\hline & & (12.48) & & & & (15.18) & \\
\hline \multirow[t]{2}{*}{7} & CIP 398203-8-2-2 & 10.00 & MR & 52 & CIP 398201-7-2-1 & 10.00 & MR \\
\hline & & (18.43) & & & & (15.18) & \\
\hline \multirow[t]{2}{*}{8} & CIP 302024-2-3-1 & 6.70 & MR & 53 & CIP 398201-15-4-1 & 30.00 & ScR \\
\hline & & (12.48) & & & & (32.99) & \\
\hline \multirow[t]{2}{*}{9} & CIP 302024-3-3-1 & 16.70 & SR & 54 & CIP 398201-5-3-1 & 16.70 & SR \\
\hline & & $(23.84)$ & & & & $(23.84)$ & \\
\hline \multirow[t]{2}{*}{10} & CIP 302024-3-4-2 & 23.30 & ScR & 55 & CIP 398201-11-6-1 & 3.30 & MR \\
\hline & & (28.77) & & & & $(6.52)$ & \\
\hline \multirow[t]{2}{*}{11} & CIP 302024-5-1-1 & 13.30 & SR & 56 & CIP 398201-2-2-1 & 10.00 & MR \\
\hline & & (21.14) & & & & (15.18) & \\
\hline \multirow[t]{2}{*}{12} & CIP 302024-6-2-1 & 10.00 & MR & 57 & CIP 304014-8-4-2 & 6.70 & MR \\
\hline & & $(15.18)$ & & & & $(12.48)$ & \\
\hline \multirow[t]{2}{*}{13} & CIP 302431-2-1-1 & 3.30 & MR & 58 & CIP 304014-2-6-1 & 3.30 & MR \\
\hline & & $(6.52)$ & & & & $(6.52)$ & \\
\hline \multirow[t]{2}{*}{14} & CIP 302431-2-3-2 & 10.00 & MR & 59 & CIP 304014-1-3-1 & 0.00 & HR \\
\hline & & $(15.18)$ & & & & $(0.57)$ & \\
\hline \multirow[t]{2}{*}{15} & CIP 302431-3-2-1 & 13.30 & SR & 60 & CIP 304014-12-2-1 & 0.00 & HR \\
\hline & & (21.14) & & & & $(0.57)$ & \\
\hline \multirow[t]{2}{*}{16} & CIP 302431-4-1-1 & 0.00 & HR & 61 & CIP 304014-5-4-1 & 6.70 & MR \\
\hline & & $(0.57)$ & & & & (12.48) & \\
\hline \multirow[t]{2}{*}{17} & CIP 302431-5-3-2 & 10.00 & MR & 62 & CIP 304014-9-2-1 & 10.00 & MR \\
\hline & & $(18.43)$ & & & & (15.18) & \\
\hline \multirow[t]{2}{*}{18} & CIP 302431-6-4-1 & 6.70 & MR & 63 & CIP 398181-9-3-1 & 26.70 & ScR \\
\hline & & $(12.48)$ & & & & $(30.98)$ & \\
\hline \multirow[t]{2}{*}{19} & CIP 304012-1-4-1 & 0.00 & HR & 64 & CIP 398181-17-2-1 & 3.30 & MR \\
\hline & & $(0.57)$ & & & & $(6.52)$ & \\
\hline \multirow[t]{2}{*}{20} & CIP 304012-6-1-1 & 3.30 & MR & 65 & CIP 398181-14-5-1 & 13.30 & SR \\
\hline & & $(6.52)$ & & & & $(21.14)$ & \\
\hline \multirow[t]{2}{*}{21} & CIP 304012-6-2-3 & 0.00 & HR & 66 & CIP 398181-7-4-1 & 13.30 & SR \\
\hline & & $(0.57)$ & & & & $(21.14)$ & \\
\hline 22 & CIP 304012-7-3-1 & 16.70 & SR & 67 & CIP 398181-10-1-1 & 0.00 & HR \\
\hline & & $(23.84)$ & & & & $(0.57)$ & \\
\hline 23 & CIP 304012-12-4-1 & 6.70 & MR & 68 & CIP 398181-16-5-1 & 6.70 & MR \\
\hline & & (12.48) & & & & (12.48) & \\
\hline 24 & CIP 304012-9-2-1 & 6.70 & MR & 69 & CIP 304124-2-5-1 & 3.30 & MR \\
\hline
\end{tabular}


Int.J.Curr.Microbiol.App.Sci (2019) 8(8): 769-776

\begin{tabular}{|c|c|c|c|c|c|c|c|}
\hline & & $(12.48)$ & & & & $(6.52)$ & \\
\hline \multirow[t]{2}{*}{25} & CIP 303405-4-2-2 & 10.00 & MR & 70 & CIP 304124-7-2-1 & 0.00 & HR \\
\hline & & $(15.18)$ & & & & $(0.57)$ & \\
\hline \multirow[t]{2}{*}{26} & CIP 303405-3-1-1 & 13.30 & SR & 71 & CIP 304124-8-3-1 & 10.00 & MR \\
\hline & & (21.14) & & & & $(15.18)$ & \\
\hline \multirow[t]{2}{*}{27} & CIP 303405-3-1-2 & 10.00 & MR & 72 & CIP 304124-1-4-1 & 10.00 & MR \\
\hline & & (18.43) & & & & (15.18) & \\
\hline \multirow[t]{2}{*}{28} & CIP 303405-8-3-1 & 6.70 & MR & 73 & CIP 304124-4-3-1 & 13.30 & SR \\
\hline & & (12.48) & & & & (21.14) & \\
\hline \multirow[t]{2}{*}{29} & CIP 303405-6-2-1 & 13.30 & SR & 74 & CIP 303139-4-4-1 & 3.30 & MR \\
\hline & & (21.14) & & & & $(6.52)$ & \\
\hline \multirow[t]{2}{*}{30} & CIP 303405-7-4-1 & 0.00 & HR & 75 & CIP 303139-9-2-1 & 13.30 & SR \\
\hline & & $(0.57)$ & & & & (21.14) & \\
\hline \multirow[t]{2}{*}{31} & CIP 303405-9-1-1 & 0.00 & HR & 76 & CIP 303139-9-4-1 & 10.00 & MR \\
\hline & & $(0.57)$ & & & & (15.18) & \\
\hline \multirow[t]{2}{*}{32} & CIP 303405-5-3-1 & 3.30 & MR & 77 & CIP 303139-2-2-1 & 3.30 & MR \\
\hline & & $(6.52)$ & & & & $(6.52)$ & \\
\hline \multirow[t]{2}{*}{33} & CIP 303408-6-3-1 & 6.70 & MR & 78 & CIP 303139-8-3-1 & 6.70 & MR \\
\hline & & (12.48) & & & & $(9.23)$ & \\
\hline \multirow[t]{2}{*}{34} & CIP 303408-8-2-2 & 13.30 & SR & 79 & CIP 303139-10-3-1 & 3.30 & MR \\
\hline & & (21.14) & & & & $(6.52)$ & \\
\hline \multirow[t]{2}{*}{35} & CIP 303408-8-3-1 & 13.30 & SR & 80 & CIP 398068-15-3-1 & 13.30 & SR \\
\hline & & (21.14) & & & & (21.14) & \\
\hline \multirow[t]{2}{*}{36} & CIP 303408-4-5-1 & 10.00 & MR & 81 & CIP 398068-15-3-2 & 6.70 & MR \\
\hline & & (18.43) & & & & (12.48) & \\
\hline \multirow[t]{2}{*}{37} & CIP 303408-2-1-1 & 10.00 & MR & 82 & CIP 398068-3-2-1 & 3.30 & MR \\
\hline & & (15.18) & & & & $(6.52)$ & \\
\hline \multirow[t]{2}{*}{38} & CIP 303408-7-3-1 & 3.30 & MR & 83 & CIP 398068-9-4-1 & 3.30 & MR \\
\hline & & $(6.52)$ & & & & $(6.52)$ & \\
\hline \multirow[t]{2}{*}{39} & CIP 303408-3-2-1 & 6.70 & MR & 84 & CIP 398068-1-5-1 & 16.70 & SR \\
\hline & & (12.48) & & & & (20.11) & \\
\hline \multirow[t]{2}{*}{40} & CIP 304102-11-1-2 & 20.00 & SR & 85 & CIP 398068-4-2-1 & 6.70 & MR \\
\hline & & (26.06) & & & & (12.48) & \\
\hline \multirow[t]{2}{*}{41} & CIP 304102-9-3-1 & 16.70 & SR & 86 & CIP 398068-7-3-1 & 13.30 & SR \\
\hline & & (23.84) & & & & $(17.89)$ & \\
\hline \multirow[t]{2}{*}{42} & CIP 304102-4-2-1 & 23.30 & ScR & 87 & KufriJawahar & 16.70 & SR \\
\hline & & $(28.77)$ & & & & $(23.84)$ & \\
\hline \multirow[t]{2}{*}{43} & CIP 304102-4-5-1 & 13.30 & SR & 88 & KufriAshoka & 6.70 & MR \\
\hline & & $(21.14)$ & & & & $(12.48)$ & \\
\hline \multirow[t]{2}{*}{44} & CIP 304102-7-1-1 & 13.30 & SR & 89 & KufriPukhraj & 3.30 & MR \\
\hline & & (21.14) & & & & $(6.52)$ & \\
\hline \multirow[t]{4}{*}{45} & CIP 304146-12-4-1 & 6.70 & MR & 90 & Kufri Surya & 3.30 & MR \\
\hline & & (12.48) & & & & $(6.52)$ & \\
\hline & & & & & $\mathrm{SEm} \pm$ & 5.149 & \\
\hline & & & & & C.D. (at $5 \%$ ) & 14.27 & \\
\hline
\end{tabular}

Average of three replications $\mathrm{MR}=$ Moderately resistant $\mathrm{SR}=$ Slightly resistant
Data in parenthesis are transformed value) MS=Moderately susceptible $\mathrm{S}=$ Susceptible
$\mathrm{HR}=$ Highly resistant

$\mathrm{ScR}=$ Scarcely resistant 
Genotypes of potato were sown in field to evaluate the resistance against leaf curl virus under natural conditions (Bagnall and Tai., 1886). All the 18 bulk population of potato showed moderate resistant reaction against leaf curl virus. In $43 \mathrm{~F} 1 \mathrm{C} 2$ clonal progenies the incidence per cent of virus was found to be zero in CIP 398203-2-1, CIP 304014-2-1 and KufriPukhraj. These progenies showed highly resistant reaction against leaf curl virus. However, 28 progenies showed moderate resistance, 7 progenies exhibited slighty resistance and 5 progenies showed scarcely resistance. Out of 90 F1C3 clonal progenies eleven progenies were found to be resistant to leaf curl virus. The resistant progenies were CIP 398203-2-2-1, CI 302431-4-1-1, CIP 304012-1-4-1, CIP 304012-6-2-3, CIP 303405-7-4-1, CIP 303405-9-1-1, CIP 304146-11-2-2, CIP 304014-1-3-1, CIP 304014-12-2-1, CIP 398181-10-1-1 and CIP 304124-7-2-1. Fifty three progenies were found with moderate resistance, 22 progenies showed slightly resistance and rest of the progenies were scarcely resistant against leaf curl virus. Pandey et al., (2006) observed that var. Kufri Chipsona-3 are moderately resistant to leaf roll virus in potato. Brown et al., (1997) also screened 12 clones with tester varieties for field resistance to potato leaf roll luteo virus. Similar results are given by Lakra (2003b), Lakra (2009), Arkorful et al., (2015), Manoj et al., (2015) and Saeed et al., (2017) for screening of potato progenies against leaf curl virus under natural field condition.

Screening against Leaf curl virus revealed that out of 151 Potato entries (18 bulk population, $43 \mathrm{~F}_{1} \mathrm{C}_{2}$ and $90 \mathrm{~F}_{1} \mathrm{C}_{3}$ progenies), most of the genotypes of clonal progenies $\left(\mathrm{F}_{1} \mathrm{C}_{2}\right.$ and $\left.\mathrm{F}_{1} \mathrm{C}_{3}\right)$ and all the genotypes of clonal bulks were found moderately resistant against leaf curl virus. Three genotypes (CIP 398203-2-1, CIP 304014-2-1 and KufriPukhraj) of $\mathrm{F}_{1} \mathrm{C}_{2}$ progenies and 11 genotypes (CIP 398203-2-2-1, CIP 302431-41-1, CIP 304012-1-4-1, CIP 304012-6-2-3, CIP 303405-7-4-1, CIP 303405-9-1-1, CIP 304146-11-2-2, CIP 304014-1-3-1, CIP 304014-12-2-1, CIP 398181-10-1-1, CIP 304124-7-2-1) of $\mathrm{F}_{1} \mathrm{C}_{3}$ progenies exhibited highly resistant reaction against leaf curl virus. Rest of the genotypes showed either slightly or scarcely resistance.

It is concluded that among 151 genotypes screened under field conditions in the present study only 14 genotypes showed highly resistance reaction against leaf curl virus. The genotypes were identified resistant to leaf curl virus disease incidence can be used for developing high yielding and disease resistant varieties in potato.

\section{References}

Arkorful, E.; Appiah, A. S. and DzahiniObiatey, H. (2015). Screening for sweet potato (Ipomoea batatas L.) Leaf curl virus (splcv) and its elimination using thermotherapy-meristem tip culture technique. The Journal of Agricultural Sciences, vol.10, pp 1-9.

Bagnall, R.H. and G.C.C. Tai. (1986). Potato leafroll virus: Evaluation of resistance in potato cultivars. Plant Dis., 70: pp 621-623.

Brown, C.R., Corsini, D., Pavek, J. and Thomas, P.E. (1997). Heritability of field resistance to potato leaf roll virus in cultivated potato. Plant-Breeding., 16(6): pp 585-588.

Garg, I. D., Kumar, S., Khurana, S. M. P. and Lakra, B. S. (2001). Association of geminivirus with potato apical leaf curl in India and its immuno-electron microscopic detection. J. Indian Potato Assoc., 28(2- 4): pp 227-232.

Khurana, S. M. P. (1999). Potato viruses and viral diseases. Technical Bull. No. 35(Revised), CPRI, Shimla, 94 p. 
Kumar, Manoj; Gupta, Anil; Singh Jagdeep; Singh, Fateh, (2015). Screening of germplasm lines against potato apical leaf curl virus disease in potato crop. International Journal of Tropical Agriculture., Vol.33 No.2 (Part III) pp. 1283-1285.

Lakra, B. S. (2003a). Potato apical leaf-curl begomovirus- symptom, appraisal of a scale and losses in potato crop. J. Indian Potato Assoc., 30(1-2): pp 119-120.

Lakra, B. S. (2003b). Effect of date of planting on whitefly population, leafcurl incidence and yield of potato cultivars. J. Indian Potato Assoc., 30(12): pp 115-116.

Lakra, B. S. (2009). Degeneration of potato cultivars due to potato apical leaf-curl virus disease. J Myco Pl Pathol., 39(3): pp 548.
Lakra, B.S. (2002). Leaf-curl: A threat to potato crop in Haryana. $\mathrm{J}$ Myco $\mathrm{Pl}$ Pathol., 32: pp 367.

Pandey, S.K., Singh, S.V., Kumar, D., Manivel, P., Marwaha, R.S., Kumar, P. and Singh, B.P. (2006). Kufri Chipsona3: A high yielding potato variety for chipping with defect free tubers. Potato $J ., 33$ (1-2): pp 26-34.

Pushkarnath (1976). Potato in sub-tropics, Orient Longman Ltd., New Delhi.

Saeed S.; Mohsan M. and Sattar A. (2017). Screening of potato (cultivar) Germplasm against Potato Leaf Roll Virus (PLRV) under natural field conditions. Advance Research Journal of Multidisciplinary Discoveries. 10.0, C-3(2017): pp 14-17.

\section{How to cite this article:}

Nirmodh Prabha, H.C. Nanda and Suresh Kumar Sharma. 2019. Identification of Promising Clones of Potato for Resistance against Leaf Curl Virus. Int.J.Curr.Microbiol.App.Sci. 8(08): 769-776. doi: https://doi.org/10.20546/ijcmas.2019.808.087 